\title{
Tools and Procedures for the ASTRI Mini-Array Calibration
}

T. Mineo, ${ }^{a, *}$ M.C. Maccarone, ${ }^{a}$ L.A. Antonelli, ${ }^{b}$ F. Ambrosino, ${ }^{b}$ P.G. Bruno, ${ }^{c}$ A. Bulgarelli, ${ }^{d}$ M. Capalbi, ${ }^{a}$ O. Catalano, ${ }^{a}$ M. Centrone, ${ }^{b}$ A.A. Compagnino, ${ }^{a}$ A. Di Paola, ${ }^{b}$ M. Faccini, ${ }^{b}$ S. Giarrusso, ${ }^{a}$ V. Giordano, ${ }^{c}$ D. Impiombato, ${ }^{a}$ S. Iovenitti, ${ }^{e}$ G. Leto, ${ }^{c}$ S. Lombardi, ${ }^{b}$ F. Lucarelli, ${ }^{b}$ D. Mollica, ${ }^{a}$ G. Pareschi, ${ }^{e}$ N. Parmiggiani, ${ }^{d}$ C. Righi, ${ }^{e}$ P. Sangiorgi, ${ }^{a}$ S. Scuderi, ${ }^{c}$ A. Segreto, ${ }^{a}$ G. Sironi, ${ }^{e}$ G. Sottile, ${ }^{a}$ R. Speziali, ${ }^{b}$ G. Tosti, $^{f}$ and L. Zampieri ${ }^{g}$ for the ASTRI Project

${ }^{a}$ INAF - IASF Palermo, via U.La Malfa 153, 90146 Palermo, Italy

${ }^{b}$ INAF - OAR, via Frascati 33, 00078 Monte Porzio Catone (RM), Italy

${ }^{c}$ INAF - OACT, Via Santa Sofia 78, 95123 Catania, Italy

dINAF - OAS, Via Piero Gobetti, 93/3, 40129 Bologna, Italy

e INAF - OAB, Via Brera, 28, 20121 Milano, Italy

${ }^{f}$ Dipartimento di Fisica e Geologia, Università di Perugia, 06123, Perugia, Italy

g INAF - OAP, Vicolo dell'Osservatorio 5, 35122 Padova, Italy

E-mail: teresa.mineo@inaf.it

The ASTRI Mini-Array (ASTRI MA) is an INAF project to construct and operate an array of Imaging Atmospheric Cherenkov Telescopes to study gamma-ray sources in the TeV range. The ASTRI MA will consist of nine double-mirror telescopes that will be installed at the Teide Astronomical Observatory, Instituto de Astrofísica de Canarias, in Tenerife (Spain). Its main scientific goal is to perform very high-energy observations of galactic and extragalactic sources with sensitivity at multi-TeV energies better than currently operating Cherenkov telescope arrays. Furthermore, the ASTRI MA telescopes will also perform intensity interferometry observations of a selected sample of bright sources being each telescope equipped with a Stellar Intensity Interferometry Instrument. The ASTRI MA requires several calibration tasks, that concern specific subsystems (i.e., optical system, Cherenkov camera, intensity interferometry instrument), the entire single telescopes and the overall array. The ASTRI MA calibration plan has the final aim to provide all the procedures and quantities necessary to correctly calibrate the scientific data. The calibration system will also serve to monitor the health of the telescopes and to contribute to the definition of good time intervals during which the observational data can be considered of high quality. Although all the ASTRI MA subsystems will be calibrated during the assembly, integration, verification and commissioning phases, some calibration tasks need to be performed periodically for maintenance and some other every observing night for monitoring instrumental parameters that can change on a short time scale. In this contribution, we present the ASTRI MA calibration plan together with methods and auxiliary equipment currently under development and testing.

$37^{\text {th }}$ International Cosmic Ray Conference (ICRC 2021)

July 12th-23rd, 2021

Online - Berlin, Germany

\footnotetext{
*Presenter
} 


\section{Introduction}

The ASTRI Mini-Array (ASTRI MA) is a project of the Italian Istituto Nazionale di Astrofisica (INAF) to construct and operate an array of nine innovative Imaging Atmospheric Cherenkov Telescopes (IACT) to study gamma-ray sources emitting at very high-energy, more specifically in the TeV spectral band [1]. The design of the ASTRI MA telescopes, with their lightened structure and the new ASTRICAM Silicon photomultipliers Cherenkov camera, is an evolution of the double-mirror ASTRI-Horn telescope (ref) successfully operating since 2014 at the INAF "M.C. Fracastoro" observing station (Serra La Nave, Mount Etna, Italy). The ASTRI-MA will be installed at the Teide Astronomical Observatory, Instituto de Astrofisica de Canarias (IAC), on Mount Teide ( $2400 \mathrm{~m}$ a.s.l.) in Tenerife (Canary Islands, Spain) and it will be operated by INAF on the basis of a host agreement with the Instituto de Astrofisica de Canarias (IAC).

The main scientific goal of the ASTRI MA is to perform very high-energy ( $\mathrm{E}>1 \mathrm{TeV}$ ) observations of galactic [2] and extragalactic [3] sources with a sensitivity at energies greater than a few $\mathrm{TeV}$ better than that reachable by the other IACTs currently in operation [4]. Furthermore, the ASTRI MA will perform a study of intensity interferometry of a selected sample of bright sources, and, for this purpose, each telescope will be equipped with a Stellar Intensity Interferometer Instrument (SI3).

The ASTRI MA requires several calibration items both for the subsystems forming the telescopes (mount, optical system, Cherenkov camera, intensity interferometry module) and for the entire array. The purpose of the calibration is to obtain all the necessary calibration coefficients to correctly calibrate the ASTRI MA scientific data, keeping all the systematic uncertainties at a level below that obtainable for the atmospheric factors. At the same time, it allows monitoring the health of the telescopes and possible degradation of their subsystems.

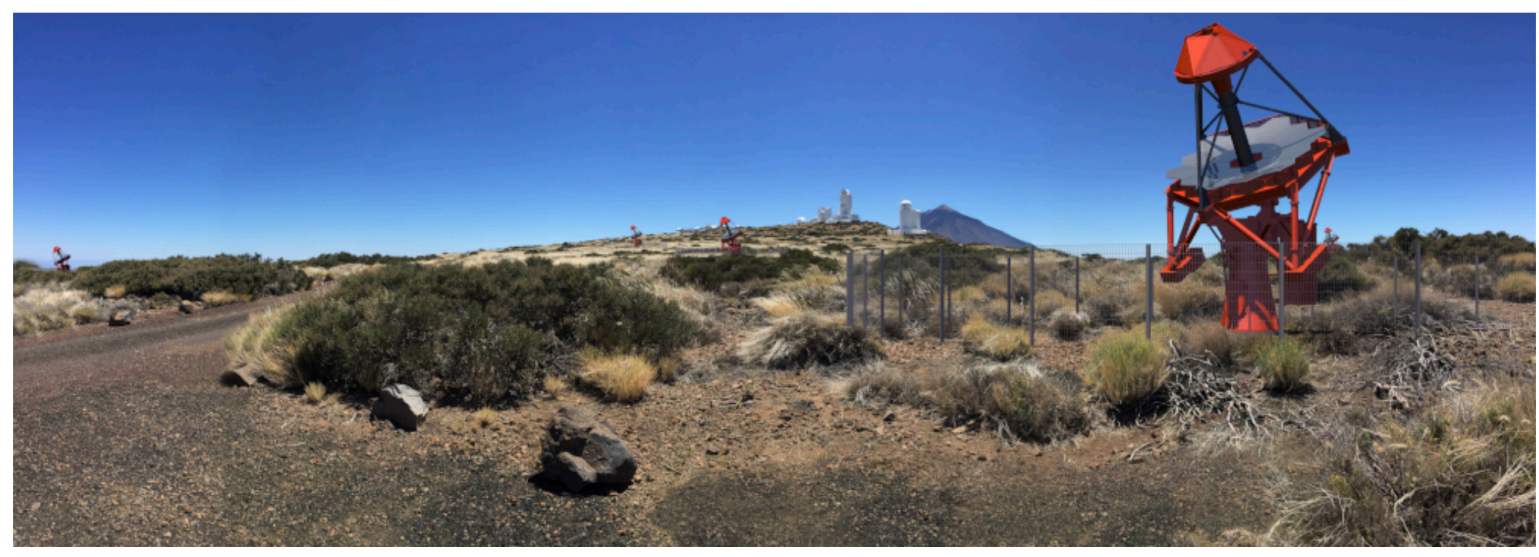

Figure 1: Artistic view of the ASTRI MA at the Teide Observatory site (Tenerife, Spain).

Although all calibrations would be fulfilled during the assembly, integration, verification and commissioning phases, some calibration tasks need to be performed periodically for monitoring purposes even at any observing night. The basic principles to be fulfilled for a successful array calibration strategy must take into account that: the calibration activities should not (or minimally) interfere with regular data taking; the sources of calibration that come for free (such as muons and air 
shower data) should be exploited as much as possible; redundancy and possibility of cross-checking should be provided in order to estimate the scale of systematic uncertainties.

The design of an overall calibration strategy is a broad band activity, which requires interaction between the hardware teams of the different telescope components and the groups involved in atmospheric monitoring, array control, data analysis and Monte Carlo simulations. In this contribution we present the methods and the auxiliary equipment currently under development and testing for the calibration of the ASTRI MA Cherenkov telescopes.

\section{The ASTRI MA Calibration Tools and Methods}

To successfully achieve the various ASTRI MA calibration items, several different measurements, data analysis methods and auxiliary devices will be used, some of which will provide the same calibration parameters. The wide number of auxiliary devices and independent analysis methods can not be considered as redundant; the comparison among the various outcomes will result in a more precise and consistent evaluation of the calibration coefficients and of their systematics.

\subsection{Calibration with observational data analysis}

The calibration methods applied directly to the acquired observational data are mainly focused on two types of data: the Variance data and the muon ring images, as explained in the following.

Variance data are acquired by each Cherenkov camera during the data taking and used for several purposes. The Variance technique is based on the statistical analysis of the signal detected by the front-end electronics whose variance is proportional to the flux impinging on the camera pixel [5]. So, the Variance technique allows us to indirectly measure the sky flux and to monitor the presence of stars in the telescope field of view (FoV). The left panel of Fig. 2 shows a 3D image of a star in the camera's FoV as observed with Variance data by ASTRI-Horn. Moreover, since the positions of the stars are known with a high accuracy, the effective pointing of the telescope can be monitored by measuring the shift between the actual positions of the stars and the nominal ones in the Variance images [6]. Furthermore, these images may reveal possible misalignment among mirror panels and check for any degradation of the optical point spread function [7].

Muon ring images are acquired by every single Cherenkov camera during normal data taking as part of hadronic air showers. The Cherenkov radiation emitted by a local muon hitting the telescope primary mirror or passing nearby creates a ring-shaped image in the camera. Such muon ring images will be used to fulfil several telescopes calibration purposes: among them, evaluation of the optical throughput and monitoring of the point spread function (PSF). The expected light intensity produced by a local muon is calculated analytically: it depends on the impact distance, the mirror radius, and the wavelength range to which the camera is sensitive. The total number of photons is reconstructed from the geometrical properties of the ring image alone and the measured intensity along the ring allows us to determine the telescope optical throughput and to monitor the photon detection efficiency of single pixels. The right panel of Fig. 2 shows an example of a muon ring observed with ASTRI-Horn and used to quantify the degradation of the mirror reflectivity [8]. 


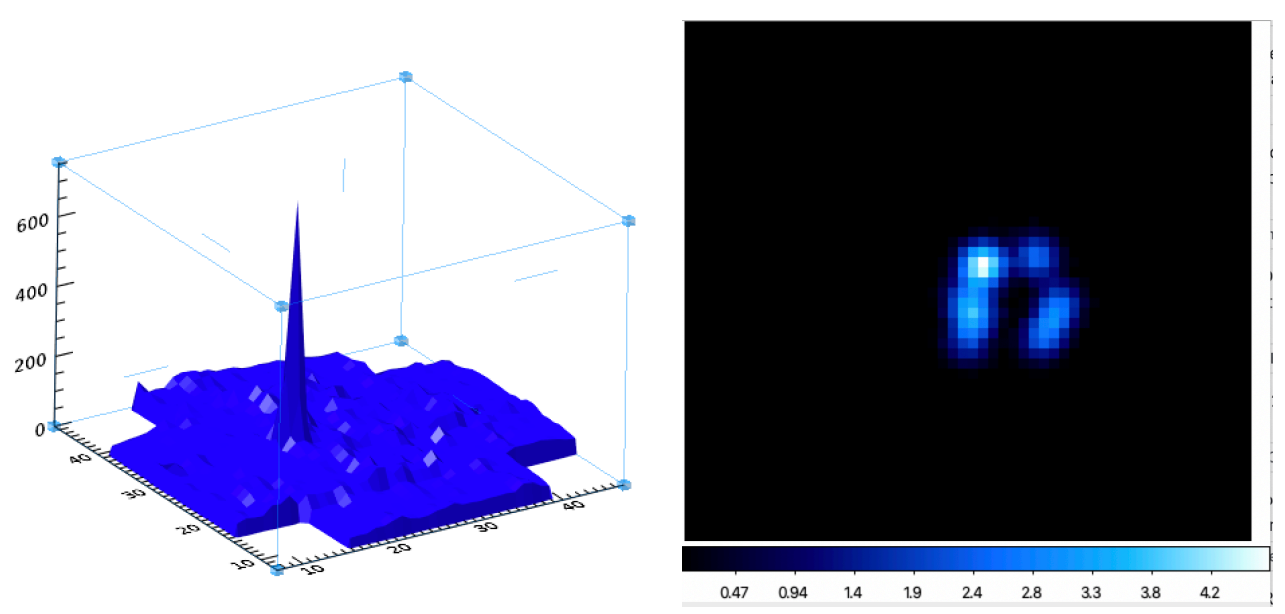

Figure 2: Left panel: 3D Variance image as observed by the ASTRI-Horn telescope, with the $\mathrm{Z}$ axis showing the level of the signal in each pixel. The peak corresponds to a star in the field of view of the telescope. Right panel: a muon ring image as observed by the ASTRI-Horn telescope.

\subsection{Calibration with auxiliary instruments}

The ASTRI MA will also benefit from auxiliary instruments specifically devoted to calibration purposes. They are listed below:

Fiber Optic Calibrator (FOC): it is an ancillary device devoted to the calibration of each pixel lodged in each ASTRICAM Cherenkov camera. A 2-mm diameter optical fiber, illuminated by blue, green, and red laser diodes, is placed in contact with the edge of the protecting window covering the focal plane detector. The light emitted from the laser diode can be continuous or pulsed and when it propagates from the optical fiber inside the protecting window it is diffused/scattered illuminating the focal plane detector uniformly;

Pointing Monitor Camera (PMC): the device [9] is hosted by each telescope to obtain an astrometric calibrated FoV of the region pointed by the telescope. The PMC, based on a CCD camera and systems of lenses, is installed on the rear of the secondary mirror support structure of the telescope. The PMC system assures a FoV of about $3^{\circ} \times 2^{\circ}$, a pixel sampling of about 7 arcsec, an astrometric accuracy of 5 arcsec over the full sky. The measured limiting magnitude is about 8 ;

UVSiPM: it is a light calibrated detector devoted to the evaluation of the local Night Sky Background (NSB). It is essentially formed by a photon detector with its front-end and data acquisitions electronics unit working in Single Photon Counting mode, and a pinhole collimator to regulate the FoV of the detector. UVSiPM is the evolution of the UVscope instrument [10] mounted on ASTRI-Horn and whose sensor is a multianode photomultiplier. Differently from its parent, the UVSiPM sensor is a single $8 \times 8$ Silicon Photo-Multiplier unit, the same model adopted in the ASTRI Cherenkov camera; this allows to evaluate the local NSB in the same wavelength range of the ASTRI camera. Only one UVSiPM unit is foreseen for the entire ASTRI MA; mounted on the external structure of one of its telescopes, and aligned with the related ASTRI Cherenkov camera, the UVSiPM will acquire data (counts/sec) contemporarily with the camera. The reduction and analysis of such data provides the evaluation of the NSB in physical units $\left.\mathrm{ph} /\left(\mathrm{m}^{2} \mathrm{~ns} \mathrm{deg}\right)^{2}\right)$ at, generally, a frequency of $1 \mathrm{~Hz}$ around the ASTRI telescope pointing direction within the UVSiPM 
FoV. This auxiliary device will be used even as a support instrument for the end-to-end absolute calibration of the ASTRI MA telescopes performed with the 'Illuminator', and for the monitoring of the NSB during the data acquisition by the SI3 instrument [11].

Illuminator: this portable ground-based device is designed to uniformly illuminate, from a certain distance, the telescope's aperture with a pulsed or continuous reference photon flux, and it will be used to perform the absolute end-to-end calibration of each telescope of the ASTRI MA. The system allows us to measure the actual spectral and temporal response of each ASTRI MA telescope at any off-axis angle, by changing the telescope pointing with respect to the Illuminator. Results will allow to obtain, for groups of few pixels, the corrective factor for the flat fielding of sky images.

Moreover, ASTRI MA is equipped with the Environmental Monitoring System, a set of devices, operating 24 hours a day, for the evaluation of the environmental conditions. Weather stations, allsky-cameras, humidity and rain sensors form the system. An essential set of the environmental parameters is sent to the Alarm System to inform the Operator of situations which could determine immediate reactions during the night to change the observations schedule [12]. All the acquired environmental data types will be archived in the proper repository and made available for the human readable weather report as well as support for the analysis of scientific observations.

\section{The calibration plan}

The ASTRI MA calibration is firstly independently performed on each telescope and on all its components (optics and camera) including the absolute calibration of the optical throughput and of the pointing precision. Once all telescopes are calibrated, the overall array calibration will be performed through the inter-calibration among the ASTRI MA telescopes and the control of the array time resolution.

\subsection{The Calibration of Individual Telescopes}

The aim of this calibration is to monitor the stability of the coefficients relative to all the telescope subsystems. These parameters will be measured during the assembly phase. However, some of them will be monitored periodically, during the regular data taking, to check the degradation of the system. In particular, the calibration of the optical system has the main purpose of assessing the status of the mirrors' reflectivity and of the optical system angular resolution. This can be performed using the analysis of either the muon ring images and the Variance data. The gain, pedestal, dark noise of each pixel will be monitored regularly with ad-hoc procedure and making use of the FOC. The pointing calibration, that aims at the correction of pointing systematic errors related to the telescope dynamics, can be performed night by night with the Variance data using the capability of the ASTRI MA Cherenkov cameras to observe stars and with the PMC mounted on each telescope. The calibration of the telescope optical throughput can be accomplished using both the Illuminator and the analysis of muon ring images.

\subsection{The array calibration}

The calibration of all individual telescopes will provide nine values of optical throughputs and pointing errors. Computing the average and the standard deviation of these values will provide the 
systematics on the overall array calibration.

At the same time, a proper inter-calibration of telescopes can be performed using two different methods: the comparison of each telescope trigger rate and the analysis of selected showers images. The trigger rates expected from each telescope should be statistically equivalent taking also into account the systematics on the calibrations of the optical throughput and of the trigger efficiency, if the telescopes work in nominal mode. Any variation from the expected rates must be investigated with opportune checks. Making use of the scientific data analysis pipeline [4] and selecting gamma-ray shower candidate events with equal impact distance for telescope pairs, allows to inter-calibrate the telescope response in terms of effective area and of basic image parameters. The inter-calibration of all telescopes is then performed considering that the couple of telescopes involved in the comparison changes randomly among the nine. Errors in the inter-calibration relatively to the systematics in the impact point determination are evaluated with dedicated ad-hoc simulations.

The calibration of the time resolution of the ASTRI Cherenkov cameras requires ad-hoc simulations to quantify the relative requirements. The procedure to calibrate the time resolution with the Cherenkov camera can be performed opportunely by selecting gamma shower or using the external trigger implemented in each camera. Making use of the scientific data analysis pipeline and selecting gamma-showers, i.e. with an impact point close to the center of the array, would allow us to inter-calibrate the trigger time in each telescope. Simulations will help to identify the appropriate parameters to be used for the selection as well as the systematic errors in the time resolution that can be achieved. An alternative method is based on the camera capability to acquire data by setting the external trigger. The correlation of the time recorded by each camera with respect to one assumed as reference allows us to determine the average shift between the clock of each camera and the reference one as well as the root-mean-square with respect to this average that is actually the timing resolution.

\section{Atmosphere Characterization}

The ASTRI MA calibration also includes the atmosphere characterisation. This is performed by making use of the data acquired by a set of devices (LIDAR, SQM, UVSiPM) that operate during the night observation period but also, in the case of LIDAR, during the daytime. The acquired data are archived in real-time in the proper repository available for the human-readable weather and atmosphere report. These data are also used in the Monte Carlo simulations production and in the data reduction and analysis. The main atmospheric parameters to be ingested in the simulation and data analysis processes are the atmospheric transmission, the atmospheric extinction profiles and the night sky background level.

The atmospheric transmission will be obtained by LIDAR data, during regular data taking, pointing to a region of the sky close to the RA-Dec coordinates under investigation. These values are sent to the Monitoring/Alarm system to decide whether or not to continue the ongoing observation.

The atmosphere extinction profiles, obtained with the LIDAR, are used to define the seasonal atmospheric models needed for the Monte Carlo simulations. In addition, daily profile obtained from data acquired at sunset before the start of the Cherenkov observations, and at dawn after the end of observations can be used to select the proper atmospheric model. 
The UVSiPM data analysis allows the evaluation of the diffuse NSB within its FoV around the ASTRI telescope pointing direction. The value of the diffuse NSB, expressed in physical units $\left[\mathrm{ph} /\left(\mathrm{m}^{2} \mathrm{~ns} \mathrm{sr}\right)\right]$ after elimination of the bright stars in the UVSiPM FoV, will be archived in a suitable repository as well as its time profile. The analysis of ASTRI-Horn and UVscope data showed that the Variance is linearly correlated to the NSB level [7]. The correlation coefficient depends on the characteristics of the two instruments and must be obtained when both of them are contemporarily operative. This allows a direct measurement of the level of NSB provided that the muon analysis gives no variation on the nominal optical throughput of the telescope.

\section{Conclusion}

The calibration in the ASTRI MA framework involves several aspects: from the relative calibration of each camera, to the corrections for the effective pointing of all telescopes, to the evaluation of the atmospheric conditions. Some tools are state-of-the-art in the current IACT systems, others have been specifically designed or optimized taking into account the ASTRI MA peculiarities and expectations. Altogether, such tools fulfill the requirements for a successful ASTRI MA calibration strategy.

\section{Acknowledgments}

This work was conducted in the context of the ASTRI Project. This work is supported by the Italian Ministry of University and Research (MUR) with funds specifically assigned to the Italian National Institute for Astrophysics (INAF). We acknowledge support from the Brazilian Funding Agency FAPESP (Grant 2013/10559-5) and from the South African Department of Science and Technology through Funding Agreement 0227/2014 for the South African Gamma-Ray Astronomy Programme. This work has been supported by H2020-ASTERICS, a project funded by the European Commission Framework Programme Horizon 2020 Research and Innovation action under grant agreement $\mathrm{n}$. 653477. IAC is supported by the Spanish Ministry of Science and Innovation (MICIU).

\section{References}

[1] L.A. Antonelli, The ASTRI mini-array at Teide Observatory, in 37th International Cosmic Ray Conference (ICRC2021), vol. This Proceeding of International Cosmic Ray Conference Series, July, 2021.

[2] A. D'Aí, Galactic Science with the ASTRI-Mini Array during the Observatory phase of the project, in 37th International Cosmic Ray Conference (ICRC2021), vol. This Proceeding of International Cosmic Ray Conference Series, July, 2021.

[3] F. Saturni, L. Antonelli, C. Arcaro, B. Balmaverde, J. Becerra Gonzaĺez, A. Caccianiga et al., Extragalactic Observatory Science with the ASTRI Mini-Array at the Observatorio del Teide, in 37th International Cosmic Ray Conference (ICRC2021), vol. This Proceeding of International Cosmic Ray Conference Series, July, 2021. 
[4] S. Lombardi, L.A. Antonelli, C. Bigongiari, M. Cardillo, S. Gallozzi, J. Green et al., Performance of the ASTRI Mini-Array at the Observatorio del Teide, in 37th International Cosmic Ray Conference (ICRC2021), vol. This Proceeding of International Cosmic Ray Conference Series, July, 2021.

[5] A. Segreto, O. Catalano, M.C. Maccarone, T. Mineo, A. La Barbera and F.T. Cta Astri Project, Calibration and monitoring of the ASTRI-Horn telescope by using the night-sky background measured by the photon-statistics ("variance") method, in 36th International Cosmic Ray Conference (ICRC2019), vol. 36 of International Cosmic Ray Conference, p. 791, July, 2019 [1909.08750].

[6] S. Iovenitti, O. Catalano, A. Segreto and T. Mineo, Effective pointing of the ASTRI-Horn telescope using the Cherenkov camera with the Variance method, in 37th International Cosmic Ray Conference (ICRC2021), vol. This Proceeding of International Cosmic Ray Conference Series, July, 2021.

[7] A.A. Compagnino, T. Mineo, M.C. Maccarone, O. Catalano, D. Impiombato and S. Giarrusso, The ASTRI-Horn telescope: comparison with the auxiliary UVscope measurements as calibration tool, in 37th International Cosmic Ray Conference (ICRC2021), vol. This Proceeding of International Cosmic Ray Conference Series, July, 2021.

[8] T. Mineo, M.C. Maccarone, A. Compagnino, M. Capalbi, O. Catalano, G. Contino et al., Muon calibration of the ASTRI-Horn telescope: preliminary results, in 36th International Cosmic Ray Conference (ICRC2019), vol. 36 of International Cosmic Ray Conference, p. 744, July, 2019 [1907.09197].

[9] E. Giro, R. Canestrari, P. Bruno, O. Catalano, D. Fugazza, N. La Palombara et al., The ASTRI-Horn telescope validation toward the production of the ASTRI Mini-Array: a proposed pathfinder for the Cherenkov Telescope Array, in Optics for EUV, X-Ray, and Gamma-Ray Astronomy IX, vol. 11119 of Society of Photo-Optical Instrumentation Engineers (SPIE) Conference Series, p. 111191E, Sept., 2019, DOI.

[10] M.C. Maccarone, G. La Rosa, O. Catalano, S. Giarrusso, A. Segreto, B. Biondo et al., UVscope and its application aboard the ASTRI-Horn telescope, Experimental Astronomy (2021) [2103.02233].

[11] L. Zampieri and et al., ASTRI Mini-Array Stellar Intensity Interferometry Conceptual Design document: ASTRI-DES-7400-001, Tech. Rep. ASTRI Collaboration (2021).

[12] N. Parmiggiani, A. Bulgarelli, L. Baroncelli, A. Addis, V. Fioretti, A. Di Piano et al., The Online Observation Quality System for the ASTRI Mini Array., in 37th International Cosmic Ray Conference (ICRC2021), vol. This Proceeding of International Cosmic Ray Conference Series, July, 2021. 